\title{
Effect of pelvic belt on the perception of difficulty and muscle activity during active straight leg raising test in pain-free subjects
}

\author{
Tadanobu Suehiro ${ }^{1, *}$, Yuri Yakushijin², Ami Nuibe ${ }^{3}$, Sakura Ishii', Chiharu Kurozumii ${ }^{1}$, Hiroshi Ishida \\ 'Department of Rehabilitation, Kawasaki University of Medical Welfare, Kurashiki, Japan \\ 2Department of Rehabilitation, Matsuyama Rehabilitation Hospital, Matsuyama, Japan \\ ${ }^{3}$ Department of Rehabilitation Medicine, Kurashiki Central Hospital, Kurashiki, Japan \\ ${ }^{4}$ Department of Rehabilitation, Saiseikai Kibi Hospital, Okayama, Japan
}

A pelvic belt decreases patient-reported perception of difficulty during the active straight leg raising (ASLR) test in individuals with pelvic girdle pain. However, the influence of a pelvic belt on the perception of difficulty during ASLR was not investigated in pain-free subjects. Therefore, this influence excluding the impact of pain is not clear. This paper aimed to clarify the effect of a pelvic belt on the perception of difficulty and muscle activity during ASLR performance in the subjective heavier side leg in pain-free subjects. Twenty pain-free female subjects participated. ASLR using the subjective heavier side leg was performed under two conditions: without and with a pelvic belt. Muscle activation of the external oblique, internal oblique, rectus abdominis, rectus femoris, and biceps femoris was measured during ASLR using a surface electromyograph. Difference in perceived difficulty in performing ASLR with and without a belt was assessed. In total, $80 \%$ of subjects had de- creased perception of difficulty using a pelvic belt during ASLR. For ASLR performed with a pelvic belt, muscle activity significantly decreased in the contralateral rectus abdominis, ipsilateral external oblique, and bilateral internal oblique $(P<0.05)$, while it significantly increased in the contralateral biceps femoris $(P<0.05)$. There were no significant differences in muscle activity of the ipsilateral rectus abdominis, contralateral external oblique, and ipsilateral rectus femoris between the two conditions $(P>0.05)$. In conclusion, using a pelvic belt can decrease the perception of difficulty during ASLR, and the pelvic belt may improve impairment of load transfer between the trunk and pelvis.

Keywords: Active straight leg raising, Pelvic belt, Electromyography, Perception of difficulty

\section{INTRODUCTION}

The stability of the lumbopelvic region and effective load transfer is obtained by the neural control subsystem, the active subsystem, and the passive subsystem (Panjabi, 1992). The sacroiliac joint stability depends on specific anatomical features, termed form closure and on muscles and ligaments crossing the sacroiliac joint, termed force closure (Snijders et al., 1993; Vleeming et al., 1990). Instability of the lumbopelvic region has relevance to lumbopelvic pain (Panjabi, 2003), and groin pain (Jansen et al., 2009). Pregnancy-related lumbopelvic pain and sports-related groin pain had a prevalence of 22\%-90\% (Albert et al., 2001; Nwuga, 1982) and 8\%-22\% (Gabbe et al., 2010; Paajanen et al., 2011), respectively.

The active straight leg raising (ASLR) test is used for the assessment of lumbopelvic stability of patients with lumbopelvic pain and groin pain (Sawle et al., 2019), muscle recruitment pattern, excessive lumbopelvic movement (Bruno et al., 2014), and patient-reported perception of difficulty (Bruno et al., 2014; Mens et al., 2002). Previous research has suggested that patients with chronic groin pain show delayed activation of the transversus abdominal muscle (Cowan et al., 2004), patients with pregnancy-related lower back pain and pelvic pain display overactivity of exter-
${ }^{*}$ Corresponding author: Tadanobu Suehiro (iD https://orcid.org/0000-0001-5525-839X Department of Rehabilitation, Faculty of Health Science and Technology, Kawasaki University of Medical Welfare, 288 Matsushima, Kurashiki 701-0193, Japan

E-mail: suehiro@mw.kawasaki-m.ac.jp

Received: March 18, 2019 / Accepted: April 17, 2019
This is an Open Access article distributed under the terms of the Creative Commons Attribution Non-Commercial License (http://creativecommons.org/licenses/by-nc/4.0/) which permits unrestricted non-commercial use, distribution, and reproduction in any medium, provided the original work is properly cited. 
nal oblique (EO) (de Groot et al., 2008), and patients with chronic pelvic girdle pain display a bracing strategy by bilateral tonic activation of the internal oblique (IO) and $\mathrm{EO}$, unlike healthy subjects (Beales et al., 2009). In addition, it has been reported that patient-reported perception of difficulty during ASLR is involved in disease severity in patients with lower back pain (Bruno et al., 2014; Mens et al., 2002).

A pelvic belt is used in individuals with instability of the sacroiliac joint and lumbopelvic region. A pelvic belt has been reported to increase sacroiliac joint stiffness (Damen et al., 2002) and to decrease pain (Arumugam et al., 2012; Jansen et al., 2009). In addition, a pelvic belt has been reported to decrease patient-reported perception of difficulty during ASLR in people with pelvic girdle pain (Mellin, 1990; Mens et al., 1999). However, the influence of a pelvic belt on perception of difficulty during ASLR was not investigated in pain-free subjects. Therefore, the influence of a pelvic belt on perception of difficulty during ASLR excluding the influence of pain is not clear.

A pelvic belt has been reported to change the muscle recruitment pattern in individuals with pelvic girdle pain (Beales et al., 2010). In addition, Hu et al. (2010) reported that in healthy subjects, activity of the lateral abdominal muscle decreased by using a pelvic belt during ASLR. However, the influence of a pelvic belt on muscle activity was not investigated in ASLR performance in the leg of the subjective heavier side of pain-free subjects. The feeling "heavy" during ASLR has been reported to indicate impairment of load transfer between the trunk and pelvis (Mens et al., 2010). Therefore, the influence of a pelvic belt on muscle ac- tivity during ASLR performance in the leg of the subjective heavier side is not clear. The aim of this study is to clarify the effect of a pelvic belt on the perception of difficulty and on muscle activity during ASLR performance in the leg of the subjective heavier side in pain-free subjects.

\section{MATERIALS AND METHODS}

\section{Participants}

Twenty pain-free female subjects participated in this study. The exclusion criteria were pregnancy, and past or present musculoskeletal, neurological, psychological, or cardiopulmonary disease. People who felt no difference in heaviness between both feet during ASLR were also excluded from the study. Before data collection, all the procedures were explained to the participants, and they signed an informed consent form. This study was approved by the Kawasaki University of Medical Welfare Research Ethics Committee (approval number: 17-099).

\section{Procedure}

Initially, the subjects performed ASLR on the left and right legs and decided which leg felt the heaviest. Subjects performed the ASLR using the leg of the subjective heavier side in this study. ASLR was performed under two conditions: without a pelvic belt and with a pelvic belt. The pelvic belt (COM-PRESSOR, OPTP Co., Minneapolis, MN, USA), which consists of four elastic bands and a main body belt, was placed below the anterior superior iliac spine (ASIS) (Jung et al., 2013). For performing ASLR, subjects

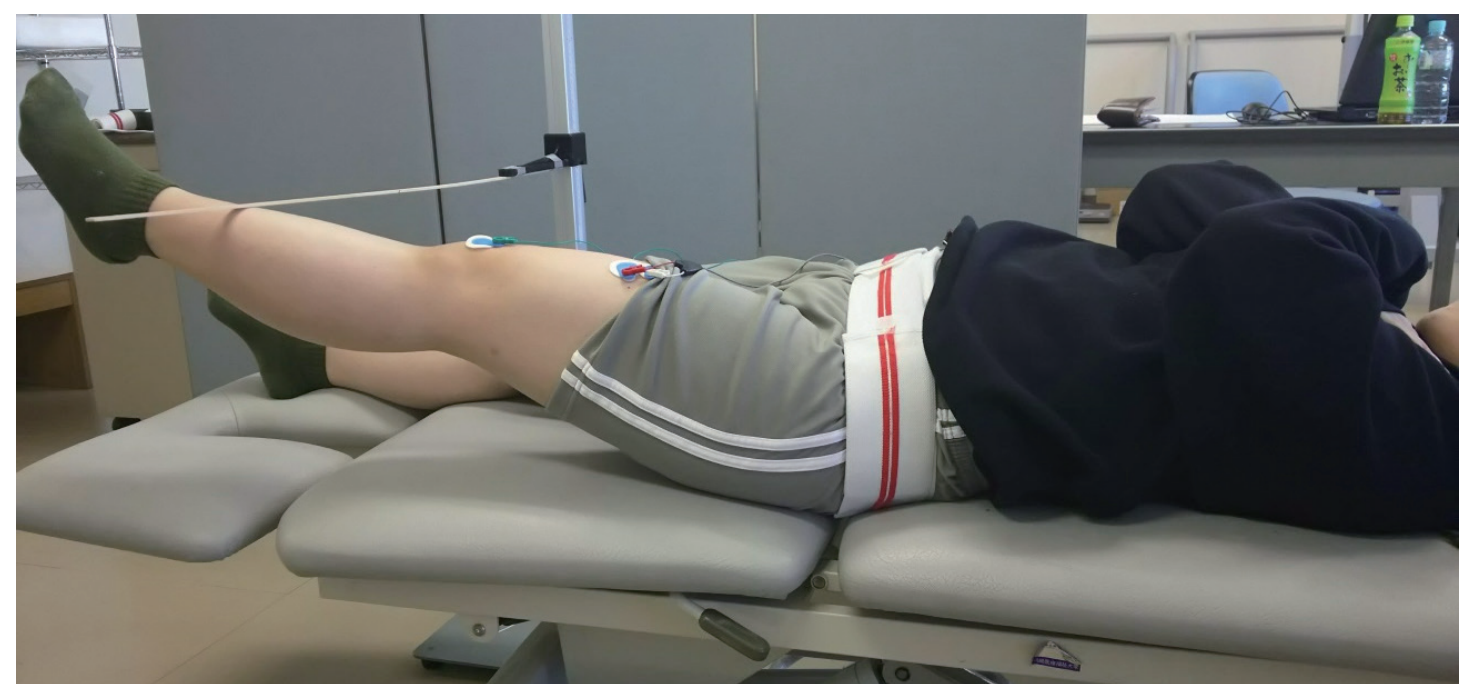

Fig. 1. The subjects raised their leg of the subjective heavier side until their leg touched the indicating bar, which was placed $20 \mathrm{~cm}$ above the bed while keeping the knee extended. The presentation of this figure was approved by the participant. 
were asked to lie supine on a treatment bed with the lower extremity, pelvis, and trunk in a straight line. Both arms were placed on their chest to prevent pushing against the bed. The subjects raised their leg of the subjective heavier side without and with a pelvic belt until their leg touched the indicating bar, which was placed $20 \mathrm{~cm}$ above the bed while maintaining the knee in extended position (Fig. 1). This position was hold for 5 sec. ASLR without and with a pelvic belt was repeated 2 times each. There was a 1-min rest between each trial. The order of measurements was randomly assigned.

\section{Electromyography}

Surface electromyography (EMG) (Vital Recorder; Kissei Comtec Co., Matsumoto, Japan) with a 1,000-Hz sampling frequency was used to measure muscle activities. The following muscles were measured: the bilateral rectus abdominis (RA: about $2-3 \mathrm{~cm}$ lateral to the umbilical region), the bilateral EO $(15 \mathrm{~cm}$ lateral to the umbilicus), the bilateral IO (2 cm medial and inferior to the ASIS), rectus femoris (RF: at $50 \%$ on the line from the superior part of the patella to the ASIS) on the leg raising side, the biceps femoris (BF: at $50 \%$ on the line between the lateral epicondyle of the tibia and the ischial tuberosity) on the non-leg raising side. The reference electrode was placed on the patella of the leg raising side. Bipolar surface electrodes (P-00-S; Ambu Inc., Ballerup, Denmark) were applied after successful skin preparation using sanding and alcohol. Each pair of electrodes was attached $2.5 \mathrm{~cm}$ apart and aligned parallel to muscle fibers. All EMG data were processed through a bandpass filter $(15-500 \mathrm{~Hz})$ and full-wave rectified. The average EMG amplitudes of the medial 3 sec of the total 5-sec duration of ASLR were determined. Mean amplitudes of the maximal voluntary isometric contraction (MVIC) were used for normalizing the average EMG amplitudes recorded during ASLR (\%MVIC). The MVIC values of each muscle were performed in the manual muscle testing positions.

\section{Subjects'scores (perception of difficulty)}

Following the completion of each test, each subject was asked to score difference in her perceived difficulty in performing ASLR

Table 1. Characteristics of the participants $(n=20)$

\begin{tabular}{lc}
\hline Characteristic & Mean \pm SD \\
\hline Age $(\mathrm{yr})$ & $21.5 \pm 0.8$ \\
Height $(\mathrm{cm})$ & $158.7 \pm 5.0$ \\
Weight $(\mathrm{kg})$ & $50.1 \pm 10.3$ \\
\hline
\end{tabular}

$\mathrm{SD}$, standard deviation. with a belt compared with performing ASLR without a belt using the following three-point scale: $-1=$ ASLR with a pelvic belt reduced the difficulty compared to ASLR without a pelvic belt; $0=$ ASLR with a pelvic belt and ASLR without a pelvic belt had the same perception of difficulty; $1=$ ASLR with a pelvic belt increased the difficulty compared to ASLR without a pelvic belt.

\section{Statistical analysis}

The Shapiro-Wilk test was used to confirm whether muscle activity data approximated a normal distribution. Values of muscle activity during ASLR were not normally distributed; therefore, the Wilcoxon signed-rank test was used to detect differences in muscle activity between ASLR without and with a pelvic belt. The $\chi^{2}$ goodness-of-fit test was used to evaluate the differences in the rate of each score of perceived difficulty. IBM SPSS Statistics ver. 22.0 (IBM Co., Armonk, NY, USA) was used for statistical analyses. Significance was set as $\alpha=0.05$.

\section{RESULTS}

Table 1 shows the physical characteristics of the participants. The scores indicating differences in their perceived difficulty in performing ASLR with a belt compared with performing ASLR without a belt are shown in Table 2. In total, $80 \%$ of subjects had decreased perception of difficulty while using a pelvic belt during ASLR, and there was a significant difference in the rate of each score of perceived difficulty $(P<0.001)$.

The activity of each muscle during ASLR without and with a pelvic belt is shown in Table 3. For ASLR performed with a pelvic belt, the muscle activity significantly decreased in the contralateral RA, ipsilateral EO, and bilateral IO $(P<0.05)$ while the muscle activity significantly increased in the contralateral $\mathrm{BF}(P<0.05)$. There were no significant differences in muscle activity of ipsilateral RA, contralateral EO, and ipsilateral RF between the two conditions $(P>0.05)$.

Table 2. Result of scores indicating difference in the perceived difficulty in performing ASLR by person with a belt compared with performing ASLR without a belt

\begin{tabular}{lc}
\hline Score & No. $(\%)$ \\
\hline-1 & $16(80.0)$ \\
0 & $3(15.0)$ \\
1 & $1(5.0)$ \\
\hline
\end{tabular}

ASLR, active straight leg raising.

There was a significant difference in the rate of each score of perceived difficulty $(P<0.001)$. 
Table 3. Electromyographic activity during active straight leg raising (\%MVIC)

\begin{tabular}{|c|c|c|c|c|c|}
\hline Variable & Without a pelvic belt & With a pelvic belt & $P$-value & Effect size $d$ & Power \\
\hline Ipsilat. RA & $5.6(4.4-8.0)$ & $4.7(3.5-6.7)$ & 0.06 & 0.47 & 0.50 \\
\hline Contralat. RA & $5.2(2.9-8.2)$ & $4.5(2.9-6.9)$ & $0.04^{*}$ & 0.51 & 0.56 \\
\hline Ipsilat. EO & $15.1(2.9-8.2)$ & $13.0(7.8-15.0)$ & $<0.01^{*}$ & 0.53 & 0.59 \\
\hline Contralat. E0 & 9.7 (7.9-21.3) & $9.7(5.0-17.6)$ & 0.07 & 0.36 & 0.32 \\
\hline Ipsilat. 10 & 12.4 (8.6-18.3) & $11.2(7.2-16.1)$ & $<0.01^{*}$ & 0.79 & 0.90 \\
\hline Contralat. 10 & $5.3(3.9-8.2)$ & $4.8(2.8-7.1)$ & $0.02^{*}$ & 0.52 & 0.57 \\
\hline Ipsilat. RF & $31.6(28.0-34.1)$ & $31.8(25.0-37.2)$ & 0.71 & 0.10 & 0.07 \\
\hline Contralat. BF & $12.2(9.6-23.1)$ & $15.1(10.5-24.7)$ & $0.02^{*}$ & 0.01 & 0.05 \\
\hline
\end{tabular}

Values are presented as median (interquartile range).

MVIC, maximal voluntary isometric contraction; Ipsilat., ipsilateral; Contralat., contralateral; RA, rectus abdominis; EO, external oblique; IO, internal oblique; RF, rectus femoris; $\mathrm{BF}$, biceps femoris.

${ }^{*} P<0.05$.

\section{DISCUSSION}

The goal of this study was to clarify the effect of a pelvic belt on the perception of difficulty and on muscle activity during ASLR performance in the leg of the subjective heavier side in pain-free subjects. In this study, the pelvic belt significantly decreased activity in the contralateral RA, ipsilateral EO, and bilateral IO during ASLR performance in the leg of the subjective heavier side, while the muscle activity significantly increased in the contralateral BF. This result resembles that of a previous study that investigated the muscle activity in healthy subjects during ASLR (Hu et al., 2012). The IO and EO activity have been theorized to act as force closure and increases the stability of the lumbopelvic region (Hu et al., 2012). Pelvic belt has been reported to increase sacroiliac joint stiffness (Damen et al., 2002). Therefore, we propose that the activity of the IO and EO, which act as force closure, decreased during ASLR using a pelvic belt because the pelvic belt substitutes as the force closure. In addition, for the ASLR, the mechanical effect of hip flexor muscles is to pull the ilium forward (Hu et al., 2010; Hu et al., 2012). Abdominal wall muscles and contralateral $\mathrm{BF}$ act to prevent anterior rotation of the pelvis and to maintain a neutral position of the pelvis against the anterior rotation of the pelvis during ASLR (Hu et al., 2010; Hu et al., 2012). It is possible that with a pelvic belt, because the contralateral RA, ipsilateral EO, and bilateral IO were less active, the contralateral $\mathrm{BF}$ was more active to prevent anterior rotation of the pelvis.

In this study, $80 \%$ of subjects showed decreased perception of difficulty by using a pelvic belt during ASLR performance in the leg of the subjective heavier side. Mens et al. (1999) have shown a decreased perception of difficulty during ASLR by using a pelvic belt in individuals with pelvic girdle pain. In this report (Mens et al., 1999), because subjects were patients with pelvic girdle pain, decreased pain and increased stability of sacroiliac joint by using a pelvic belt might have been decreasing the perception of difficulty during ASLR. In this study, however, since the subjects were painfree, decreased perception of difficulty during ASLR was not caused by a change in the degree of pain. We believe that change in the muscle recruitment pattern and increasing stability of the sacroiliac joint by using a pelvic belt may cause decreasing perception of difficulty during ASLR.

This research has some limitations. We did not measure the activation of transversus abdominis and pelvic floor muscles. In addition, we did not measure pelvic motion when recording the effect of muscle activity with a pelvic belt. Therefore, pelvic motion during ASLR using a pelvic belt remains unclear. The activity of the transversus abdominis, pelvic floor muscles, and pelvic motion during ASLR performance in the leg of the subjective heavier side should be evaluated in future investigations.

This study suggests that using a pelvic belt can decrease the perception of difficulty during ASLR and that the pelvic belt may improve impairment of load transfer between the trunk and pelvis.

\section{CONFLICT OF INTEREST}

No potential conflict of interest relevant to this article was reported.

\section{REFERENCES}

Albert H, Godskesen M, Westergaard J. Prognosis in four syndromes of pregnancy-related pelvic pain. Acta Obstet Gynecol Scand 2001;80: 
505-510.

Arumugam A, Milosavljevic S, Woodley S, Sole G. Effects of external pelvic compression on form closure, force closure, and neuromotor control of the lumbopelvic spine--a systematic review. Man Ther 2012;17: 275-284.

Beales DJ, O'Sullivan PB, Briffa NK. Motor control patterns during an active straight leg raise in chronic pelvic girdle pain subjects. Spine (Phila Pa 1976) 2009;34:861-870.

Beales DJ, O'Sullivan PB, Briffa NK. The effects of manual pelvic compression on trunk motor control during an active straight leg raise in chronic pelvic girdle pain subjects. Man Ther 2010;15:190-199.

Bruno PA, Goertzen DA, Millar DP. Patient-reported perception of difficulty as a clinical indicator of dysfunctional neuromuscular control during the prone hip extension test and active straight leg raise test. Man Ther 2014;19:602-607.

Cowan SM, Schache AG, Brukner P, Bennell KL, Hodges PW, Coburn P, Crossley KM. Delayed onset of transversus abdominus in long-standing groin pain. Med Sci Sports Exerc 2004;36:2040-2045.

Damen L, Spoor CW, Snijders CJ, Stam HJ. Does a pelvic belt influence sacroiliac joint laxity? Clin Biomech (Bristol, Avon) 2002;17:495-498.

de Groot M, Pool-Goudzwaard AL, Spoor CW, Snijders CJ. The active straight leg raising test (ASLR) in pregnant women: differences in muscle activity and force between patients and healthy subjects. Man Ther 2008;13:68-74.

Gabbe BJ, Bailey M, Cook JL, Makdissi M, Scase E, Ames N, Wood T, McNeil JJ, Orchard JW. The association between hip and groin injuries in the elite junior football years and injuries sustained during elite senior competition. Br J Sports Med 2010;44:799-802.

Hu H, Meijer OG, Hodges PW, Bruijn SM, Strijers RL, Nanayakkara PW, van Royen BJ, Wu W, Xia C, van Dieën JH. Understanding the Active Straight Leg Raise (ASLR): an electromyographic study in healthy subjects. Man Ther 2012;17:531-537.

Hu H, Meijer OG, van Dieën JH, Hodges PW, Bruijn SM, Strijers RL, Nanayakkara PW, van Royen BJ, Wu W, Xia C. Muscle activity during the active straight leg raise (ASLR), and the effects of a pelvic belt on the ASLR and on treadmill walking. J Biomech 2010;43:532-539.

Jansen JA, Mens JM, Backx FJ, Stam HJ. Changes in abdominal muscle thickness measured by ultrasound are not associated with recovery in athletes with longstanding groin pain associated with resisted hip ad- duction. J Orthop Sports Phys Ther 2009;39:724-732.

Jung HS, Jeon HS, Oh DW, Kwon OY. Effect of the pelvic compression belt on the hip extensor activation patterns of sacroiliac joint pain patients during one-leg standing: a pilot study. Man Ther 2013;18:143148.

Mellin G. Decreased joint and spinal mobility associated with low back pain in young adults. J Spinal Disord 1990;3:238-243.

Mens JM, Pool-Goudzwaard A, Beekmans RE, Tijhuis MT. Relation between subjective and objective scores on the active straight leg raising test. Spine (Phila Pa 1976) 2010;35:336-339.

Mens JM, Vleeming A, Snijders CJ, Koes BW, Stam HJ. Validity of the active straight leg raise test for measuring disease severity in patients with posterior pelvic pain after pregnancy. Spine (Phila Pa 1976) 2002; 27:196-200.

Mens JM, Vleeming A, Snijders CJ, Stam HJ, Ginai AZ. The active straight leg raising test and mobility of the pelvic joints. Eur Spine J 1999;8:468473.

Nwuga VC. Pregnancy and back pain among upper class nigerian women. Aust J Physiother 1982;28:8-11.

Paajanen H, Ristolainen L, Turunen H, Kujala UM. Prevalence and etiological factors of sport-related groin injuries in top-level soccer compared to non-contact sports. Arch Orthop Trauma Surg 2011;131:261266.

Panjabi MM. The stabilizing system of the spine. Part I. Function, dysfunction, adaptation, and enhancement. J Spinal Disord 1992;5:383389.

Panjabi MM. Clinical spinal instability and low back pain. J Electromyogr Kinesiol 2003;13:371-379.

Sawle L, Freeman J, Marsden J. A Pilot RCT investigating the effects of targeted compression on athletes with pelvic/groin pain. J Sport Rehabil 2019;28:133-143.

Snijders CJ, Vleeming A, Stoeckart R. Transfer of lumbosacral load to iliac bones and legs Part 1: Biomechanics of self-bracing of the sacroiliac joints and its significance for treatment and exercise. Clin Biomech (Bristol, Avon) 1993;8:285-294

Vleeming A, Stoeckart R, Volkers AC, Snijders CJ. Relation between form and function in the sacroiliac joint. Part I: Clinical anatomical aspects. Spine (Phila Pa 1976) 1990;15:130-132. 